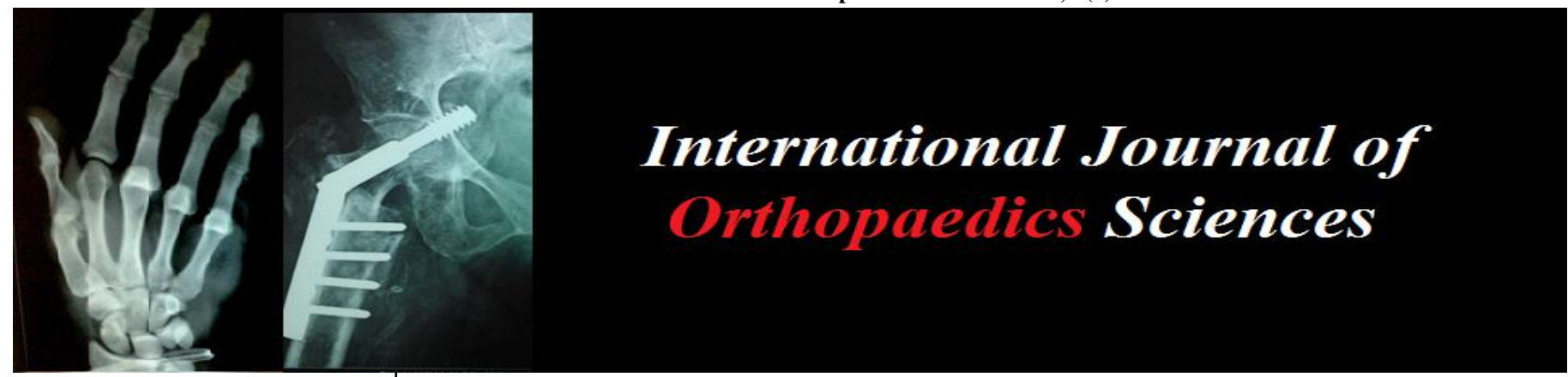

ISSN: $2395-1958$

IJOS 2018; 4(1): 886-888

(C) 2018 IJOS

www.orthopaper.com

Received: xx-11-2017

Accepted: xx-12-2017

Misbah Mehraj

Government Medical College,

Jammu and Kashmir, India

\section{Iqra Shah}

Shri Guru Ram Rai Institute of

Medical \& Health Sciences,

Uttarakhand, India

\section{Effects of intramedullary nail removal after tibial fracture repair: A prospective study}

\author{
Misbah Mehraj and Iqra Shah
}

DOI: https://doi.org/10.22271/ortho.2018.v4.i1m.128

\section{Abstract}

Introduction: Tibial fractures are the most common long bone fracture. The standard of care for the treatment of diaphyseal tibial fractures is an intramedullary nail (IMN). Implant removal is one of the most common procedures in bone and joint surgery, and criteria for implant removal are typically left to the treating surgeon. We conducted a prospective study to investigate effects of intramedullary nail removal after tibial fracture union.

Methods: Sixty patients at our hospital were enrolled in a prospective study and divided into moderate/severe knee pain (visual analog scale (VAS) - 4) and mild/no knee pain (VAS < 4) groups after interlocking intramedullary nailing. Variables studied included the distance from the tip of the nail to the tibial plateau and the front of the tibia on a normalized lateral X-ray, the VAS score of knee and ankle pain, the range of motion of the knee and ankle, and Johner-Wruhs criteria before, 6 weeks after operation, and at the last follow-up.

Results: Fifty-seven patients were followed for a mean of $8.4(2-17)$ months. In patients with moderate or severe knee pain intramedullary nail removal led to significant pain reductions $(\mathrm{p}<0.05)$. A significantly shorter distance from the tip of the nail to the tibial plateau $(<10 \mathrm{~mm})$ and the anterior border of tibia $(<6 \mathrm{~mm})$ was found in the 24 patients with moderate or severe knee pain.

Conclusion: For patients complaining knee pain after interlocking intramedullary nailing of tibial fractures, especially with a short distance from the tip of the nail tail to the tibial plateau $(<10 \mathrm{~mm})$ and the anterior border of the tibia $(<6 \mathrm{~mm})$ removal of the intramedullary nails relieved the pain significantly.

Keywords: Tibial fractures, intramedullary nail, knee pain, VAS scores

\section{Introduction}

Tibial fractures are the most common long bone fracture ${ }^{[1]}$. The method of fracture treatment depends on the characteristics of the fracture and on surgeon preference. Currently methods used include casting, plate fixation, external fixation and intramedullary nailing. For displaced diaphyseal tibial fractures, intramedullary nails (IMNs) have become the treatment of choice [2]. IMN treatment provides a high rate of union and a decreased incidence of malunion and joint stiffness, compared with other treatments ${ }^{[2,3]}$.

Within the literature, previously listed criteria for implant removal include symptomatic hardware, skeletally immature patients, broken hardware, compromised skin, nonunion, malunion, infection, fear of carcinogenesis, peri implant failure, prevention of post union stress-shielding, prevention of future bacterial colonization, avoidance of difficult surgery owing to the potential for refracture or implant failure and the possibility that removal will improve functional outcome ${ }^{[4-8]}$.

Currently, there is little consensus among orthopedic surgeons regarding criteria for tibial IMN removal post union. Most orthopedic surgeons rely on patient symptoms, especially knee and/or leg pain, to determine whether or not the implant should be removed after healing. Little evidence exists in the literature regarding the efficacy or characteristics of patients who request IMN removal. Some authors argue for an improvement in symptomatology after removal, whereas others have not found any significant improvement. We designed a prospective study to explore the indications for and complications of intramedullary nail removal after tibial fracture repair using interlocking intramedullary nails. 


\section{Material and Methods}

Sixty patients who had received interlocking intramedullary nailing at Government Medical College Jammu between May 2014 and May 2016 were included in our study. Inclusion criteria included tibial or tibiofibular fracture treated with interlocking intramedullary nails. Exclusion criteria were: (1) ipsilateral femur, tibial plateau, or ankle fracture; (2) limited range of lower limb motion or paraplegia; (3) knee ligament injury or presence of osteoarthritis; (4) experiencing trauma following nail removal.

\section{Surgical Method}

All patients were placed in the supine position and received epidural or spinal anesthesia. After exsanguination, the two distal interlocking pins and one proximal pin were first removed using the original incision. Then, after exposing the tibial slope, the last proximal pin was taken out by attaching the puller to the end of the intramedullary nail and removing the intramedullary nail using a sliding hammer. Drainage was routinely placed, and it was removed 1 day postoperatively. The incision was closed, and pressure dressing was placed on the incision with an elastic bandage. Patients were instructed to do flexion-extension exercises in bed and partial weightbearing exercises 3 days after the operation.

\section{Variables Measured}

Radiographic parameters: Following variables were measured on lateral X-ray (a) The distance from the tip of the nail to the tibial plateau; (b) The distance from the tip of the nail to the anterior border of the tibia.

Pain scores: Visual analog scale (VAS) was used to assess pain in the knee and ankle before the operation, 6 weeks after the operation, and at the last follow-up.

Range of motion: The range of motion of the knee and ankle was measured before the operation, 6 weeks after the operation, and at the last follow-up.

Functional scores: Johner-Wruhs criteria were used to assess the therapeutic effect for patients compared to before the operation, at 6 weeks after operation and at the last follow-up.

\section{Results}

A total of 60 patients were enrolled in the study: 34 males and 26 females; mean age 30.2 (24-59) years. The mean interval between admission to hospital and surgery was 5.6 (1-8) days. Forty-eight fractures were treated by the transtendinous approach (TTA) and 12 by the paratendinous (PTA).The diameter of the nail averaged $9.7(8-10) \mathrm{mm}$. The nail was removed at an average of 19.5 (12-30) months postoperatively. Fifty-seven patient were followed up for a mean of 8.4 (2-17) months. All wounds healed, and no postoperative infection or fractures occurred after interlocking intramedullary nail removal. Twenty-four of 57 patients reported moderate to severe knee pain (VAS -4). In this group of patients, the distances from the tip of the nail to the tibial plateau and the anterior border of the tibia were $8.2+5.9 \mathrm{~mm}$ and $3.2+3.5 \mathrm{~mm}$, respectively. The same distances were 11.5 $+5.3 \mathrm{~mm}$ and $5.5+4.2 \mathrm{~mm}$, respectively, in the other 33 patients, who reported mild or no knee pain (VAS $<4$ ). The VAS value for knee pain among the 57 patients decreased significantly from $3.4+2.1$ before the operation to $2.5+1.4$, 6 weeks after the operation and $2.4+1.3$ at the last follow-up. Intramedullary nail removal in the 24 patients with moderate to severe knee pain led to pain relief in 18 (75\%), to pain increase in $0(0 \%)$ and no change in $6(25 \%)$ cases. Three of five patients with limited range of knee motion showed improvement at the last follow-up.

\section{Discussion}

There is still some controversy as to whether or not the internal fixation should be removed after bone fracture repair. Busam et al. ${ }^{[9]}$ suggested that the internal fixation should not be removed, because it is associated with a high rate of complications, such as infection, removal failure, and refracture. In our study, we found a significant reduction in knee pain VAS scores after removal of the intramedullary nail, which suggests that patients complaining of knee pain would benefit from removal of the intramedullary nail. However, no significant differences in ankle pain VAS scores, range of knee and ankle motion, and Johner-Wruhs criteria were found at 6 weeks postoperatively, when compared with those before the operation. Further analysis has revealed that the incidence of knee pain was significantly greater in patients with a short distance from the tip of the nail to the tibial plateau $(<10 \mathrm{~mm})$ and anterior border of the tibia $(<6 \mathrm{~mm})$, indicating that the distance from the tip of the nail to the tibial plateau and the anterior border of the tibia played an important role in knee pain. Based on our experience, we posit that the knee pain may be due to the excessive length of the intramedullary nail used in the primary surgery. Specifically, the protruding tip of the nail can easily irritate the surrounding soft tissue, thereby resulting in knee pain. In addition, we found that the tip of the nail did not often enter the knee joint cavity, so the range of knee motion was generally not limited. For the 24 patients with knee pain VAS $>3$, we compared the VAS value at 6 weeks post operatively and at last follow-up with that before the operation, and as might be expected, the VAS value was significantly lower at both time points. In fact, similar results were reported by Bhattacharyya et al. ${ }^{[10]}$ who found that the incidence of pain was significantly less when the distance from the tip of the nail to the tibial plateau and the anterior border of the tibia were both above $1.25 \mathrm{~cm}$. They also pointed out that having the appropriate depth of the tip of the nail below the bone cortex would make it easier to remove the nail in the future. Postoperative knee pain is a common complication with interlocking intramedullary nails. For example, Leliveld and Verhofstad ${ }^{[11]}$ reported an incidence of $38 \%$ (27/71) for knee joint chronic pain. In our study, 24 of 57 patients suffered from moderate to severe knee pain (VAS -4), and the VAS score decreased significantly at 6 weeks postoperatively and at the last follow-up. However, removal of the intramedullary nail did not relieve the knee pain in all of these patients, and some patients without preoperative pain developed knee pain postoperatively. Therefore, the distance from the tip of the nail to tibial plateau or the anterior border of the tibia was not the only factor contributing to knee pain. Labronici et al. ${ }^{[12]}$ performed CT scans on 30 patients treated with interlocking intramedullary nails who had complained of knee pain postoperatively, and the results showed that the proximal interlocking pin entered the tibiofibular joint in 20 of these patients, which may be the iatrogenic cause of knee pain. In addition, Hemigou and Cohen [13] have shown that postoperative knee pain correlated with damage to the internal structure of the knee joint, including the nerves and the fat pad under the patella. There was a slope between the tibial tubercle and the anterior border of the tibial plateau, with transverse ligaments connecting the anterior edge of the medial and lateral meniscus on this slope, and a so-called safety zone for inserting the intramedullary nail existed on this slope. The anterior border of the tibial plateau, the 
transverse ligament, and the anterior horn of the meniscus were easily damaged if the entry point of the nail was beyond the safety zone. Alsousou et al. ${ }^{[14]}$ have confirmed that adhesion in the patellar tendon structure could lead to postoperative knee pain. We think that this finding could explain the new knee pain experienced by some patients in our study after removal of the intramedullary nail. Furthermore, Vaisto et al. ${ }^{[15]}$ conducted an ultrasound study of the patellar tendon after tibial nailing to compare the changes between patients with and without anterior knee pain, and the results of this study suggest that the two different approaches (TTA and PTA) did not make any difference in this regard. Therefore, in order to reduce the incidence of knee pain postoperatively in patients treated with intramedullary nails, the so-called safety zone should be identified, and an intramedullary nail with the appropriate length should be selected.

\section{Conclusion}

Our results show that removal of the intramedullary nail resulted in significant pain relief in patients complaining of knee pain after interlocking intramedullary nailing of tibial fractures, especially in those with a short distance between the tip of the nail and the tibial plateau $(<10 \mathrm{~mm})$ or the anterior border of the tibia $(<6 \mathrm{~mm})$ on normalized lateral X-rays. Close proximity of the tip of the intramedullary nail to these anatomical structures may be considered to be an indication for intramedullary nail removal after tibial fracture repair using interlocking intramedullary nails.

\section{References}

1. Praemer A, Furner S, Rice DP. Musculoskeletal conditions in the United States. Rosemont (IL): American Academy of Orthopaedic Surgeons, 1992.

2. Finkemeier CF, Schmidt AH, Kyle RF, et al. A prospective randomized study of intramedullary nails inserted with and without reaming for the treatment of open and closed fractures of the tibial shaft. J Orthop Trauma. 2000; 14:187-93.

3. Watson JT. Current concepts review. Treatment of unstable fractures of the shaft of the tibia. J Bone Joint Surg Am. 1994; 76:1575-84.

4. Muller ME, Allgower M, Schneider R, et al. Manual of internal fixation: techniques recommended by the AOASIF Group. New York: Springer Verlag, 1979.

5. Brown RM, Wheelwright EF, Chalmers J. Removal of metal implants after fracture surgery: indications and complications. JR Coll Surg Edinb. 1993; 38:96-100.

6. Browner B, Edwards C. The science and practice of intramedullary nailing. Philadelphia: Lea and Febiger, 1987.

7. Busam ML, Esther RJ, Obremskey WT. Hardware removal: indications and expectations. J Am Acad Orthop Surg. 2006; 14:113-20.

8. Toms AD, Morgan-Jones RL, Spencer-Jones R. Intramedullary femoral nailing: removing the nail improves subjective outcome Injury. 2002; 33:247-9.

9. Busam ML, Esther RJ, Obremskey WT. Hardware removal: indications and expectations. J Am Acad Orthop Surg. 2006; 14:113-120.

10. Bhattacharyya T, Seng K, Nassif NA, et al. Knee pain after tibial nailing: the role of nail prominence. Clin Orthop Relat Res. 2006; 449:303-307.

11. Leliveld MS, Verhofstad MH. Injury to the infrapatellar branch of the saphenous nerve, a possible cause for anterior knee pain after tibial nailing? Injury. 2012; 43:779-783.

12. Labronici PJ, Santos Pires RE, Franco JS, et al. Recommendations for avoiding knee pain after intramedullary nailing of tibial shaft fractures. Patient Saf Surg. 2011; 5:31.

13. Hernigou P, Cohen D. Proximal entry for intramedullary nailing of the tibia. The risk of unrecognised articular damage. J Bone Joint Surg Br. 2000; 82:33-41.

14. Alsousou J, McDonnell SM, Elo J. A simple cost effective technique for soft tissue protection during intramedullary nailing of the tibia. Acta Orthop Belg. 2010; 76:827-829.

15. Vaisto O, Toivanen J, Paakkala T, et al. Anterior knee pain after intramedullary nailing of a tibial shaft fracture: an ultrasound study of the patellar tendons of 36 patients. J Orthop Trauma. 2005; 19:311-316. 